LMU-17/93

\title{
Top Condensation without Fine-Tuning
}

\author{
Andreas Blumhoferf \\ Sektion Physik \\ Ludwig-Maximilians-Universität München \\ Theresienstr.37, D-80333 München
}

\begin{abstract}
Quadratic divergencies which lead to the usual fine-tuning or hierarchy problem are discussed in top condensation models. As in the Standard Model a cancellation of quadratic divergencies is not possible without the boson contributions in the radiative corrections which are absent in lowest order of an $1 / N_{c}$-expansion. To deal with the cancellation of quadratic divergencies we propose therefore an expansion in the flavor degrees of freedom. In leading order we find the remarkable result that quadratic divergencies automatically disappear.
\end{abstract}

${ }^{*}$ Email: AB at HEP.PHYSIK.UNI-MUENCHEN.DE 


\section{Introduction}

Today the Standard Model is in good agreement with experiment. Even though the Higgs sector is not yet tested. Due to theoretical problems and from an aesthetical point of view it is often argued that the Standard Model Higgs field cannot be the whole story explaining the symmetry breaking of the electroweak interaction. The main criticism are the quadratic divergencies which lead to an unnatural big difference between bare and renormalized quantities if the Higgs sector is valid up to the Grand Unification scale. This agrees with 't Hoofts naturalness principle [1]: A hierarchy problem appears, if there is no extra symmetry in the case that the Higgs mass goes to zero.

In the Standard Model quadratic divergencies can be seen in the radiative corrections of the Higgs tadpoles and self-energies. At the one-loop-level all massive fermions and bosons contribute with statistically induced opposite sign

$$
\left(4 m_{t}^{2}-M_{H}^{2}-M_{Z}^{2}-2 M_{W}^{2}\right) \Lambda^{2},
$$

where light fermion masses are ignored. Therefore the Higgs sector would be natural, if fermionic and bosonic parts would cancel each other. This happens for example in supersymmetric theories due to superpartners In the Standard Model, however, eq. (1) does not automatically vanish. To avoid quadratic divergencies one must postulate eq. (1) to be zero, which is the so-called Veltman condition [3]:

$$
4 m_{t}^{2}=M_{H}^{2}+M_{Z}^{2}+2 M_{W}^{2} .
$$

If $\Lambda$ is very big as suggested above, the vanishing of eq. (1) needs an extreme fine-tuning, which means that the condition eq. (2) must be fullfilled very precisely. So far no mechanism or extra symmetry was found leading in a natural way to the mass relation eq. (2). Further more the Veltman condition is regularization scheme dependent, which can for example be seen by using different cutoffs for different particles. Nevertheless the Veltman condition eq. (2) succeeded in predicting a top mass bigger than $\sqrt{M_{Z}^{2}+2 M_{W}^{2}} / 2=73 \mathrm{GeV}$ and becomes popular with the increase of the experimental lower bound on the top mass.

For a heavy Higgs and top mass eq. (2) further degenerates to

$$
M_{H} \approx 2 m_{t},
$$

which suggests that the Higgs boson could be a $\bar{t} t$-boundstate. It is therefore interesting to ask if top condensation provides a cancellation mechanism for quadratic divergencies.

*For a review see [2]. 
Keeping Supersymmetry in mind top and Higgs might play the role of superpartners in eq. (11). The term "Quasi-Supersymmetry" was therefore introduced by Nambu [4].

But for top condensation models the Higgs boson is a dynamical object and top and Higgs mass are determined by a complicated system of Schwinger-Dyson-equations. It seems therefore hard to believe that both masses are automatically arranged in such a way that eq. (2) is fullfilled. Even more there is no cancellation at all in leading order $1 / N_{c}$ due to the missing next-to-leading order Higgs and Goldstone boson graphs. To include them one also has to expand in the flavor degrees of freedom, which we will propose in the second part. In contrast to the corresponding Standard Model graphs they get additional factors leading to the remarkable result that the quadratic divergencies automatically disappear independent of the values of top and Higgs mass. In the third part we show further that the cancellation demands a special group structure of strong and weak interactions which is as an interesting fact fullfilled by the Standard Model. In the fourth part we discuss the consequences of the missing quadratic divergencies for the gap equation and the scale of the new interaction.

\section{Flavor expansion in top condensation}

We start with the minimal top condensation model first discussed by W. A. Bardeen, C. T. Hill and M. Lindner [5]. It has no Higgs sector but includes an additional fourfermion-interaction

$$
\mathcal{L}_{I}=\frac{G}{N_{c}}\left(\overline{\psi_{L}} t_{R}\right)\left(\overline{t_{R}} \psi_{L}\right) ; \quad \psi_{L}=\left(\begin{array}{c}
t_{L} \\
b_{L}
\end{array}\right)
$$

But we keep in mind that $\mathcal{L}_{I}$ is only an effective low energy description of some not specified renormalizable interaction 1 . Otherwise higher dimensional operators with arbitrary factors can appear leading to arbitrary values for top and Higgs mass [7].

In terms of auxiliary fields $H, G^{0}$ and $G^{ \pm}$the interaction has the form

$$
\begin{aligned}
\mathcal{L}_{I}= & \frac{1}{2} \frac{g_{t}^{2} N_{c}}{G}\left(H H+G^{0} G^{0}+2 G^{+} G^{-}\right) \\
& -\frac{g_{t}}{\sqrt{2}} H \bar{t} t+i \frac{g_{t}}{\sqrt{2}} G^{0} \bar{t} \gamma^{5} t+g_{t}\left(G^{+} \bar{t} L b+G^{-} \bar{b} R t\right),
\end{aligned}
$$

where $L=\left(1-\gamma^{5}\right) / 2$ and $R=\left(1+\gamma^{5}\right) / 2$. These fields are arranged in such a way that

${ }^{*}$ For special models see [6]. 
the usual Higgs and Goldstone boson couplings to the fermions of the third generation in the Standard Model appear.

The top mass is now generated by the gap equation (see Fig. 1) which gives in lowest order $1 / N_{c}$ :

$$
1=\frac{2 G}{(4 \pi)^{2}}\left(\Lambda^{2}-m_{t}^{2} \ln \frac{\Lambda^{2}}{m_{t}^{2}}\right) .
$$

Summing up the geometrical series of the boson vacuum polarisation of the different auxiliary fields (Fig. 2) and using eq. (6) one gets propagating boundstates which were introduced above as auxiliary fields:

$$
\begin{aligned}
D_{H} & =\zeta^{-1} \frac{i}{p^{2}-4 m_{t}^{2}} \\
D_{G^{0}} & =\zeta^{-1} \frac{i}{p^{2}} \\
D_{G^{ \pm}} & =\zeta^{-1} \frac{i}{p^{2}}
\end{aligned}
$$

with

$$
\zeta=\frac{N_{c} g_{t}^{2}}{(4 \pi)^{2}} \ln \frac{\Lambda^{2}}{p^{2}} .
$$

where we have neglected finite terms. As expected the Higgs mass becomes twice the top mass, which was one of the motivations for top condensation by comparison with eq. (3). The tadpole graph in Fig. 1 is quadratically divergent and corresponds to the same graph in the Standard Model, which yields the first contribution of eq. (1) with one exception: The Higgs in Fig. 1 is the non propagating auxiliary field, which means that the Higgs exchange of the tadpole is only a four-fermion-vertex.

One might wonder why there is no Higgs and Goldstone boson tadpole in Fig. 1 with regard to the second contribution in eq. (四), which is essential for a possible compensation of quadratic divergencies. But such graphs would be of higher order in $1 / N_{c}$. Nevertheless motivated by the Standard Model one has to expect that the boson tadpoles are of the same order of magnitude as the top tadpole even if there is no exact cancellation. This shows that for quadratic divergencies the $1 / N_{c}$-series unfortunately is a bad expansion in spite of the consistency with regard to symmetries.

Interestingly the boson contribution increases with the number of Goldstone bosons and is therefore bigger than in a pure Nambu-Jona-Lasinio model [8] containing a U(1) symmetry. 
That is, the number of Goldstone bosons depends on the number of particles in the weak multiplet and therefore on the group structure of the weak interaction. It seems therefore resonable to choose the group dimension as an additional expansion parameter. We call this a "flavor expansion" from now on. For this purpose we generalize the weak $S U(2)_{L}$ symmetry $S U(2)_{L} \longrightarrow S U\left(N_{L}\right)_{L}$. The left-handed doublet $\psi_{L}$ becomes then:

$$
\psi_{L}=\left(\begin{array}{c}
t_{L} \\
b_{1, L} \\
\vdots \\
b_{N_{L}-1, L}
\end{array}\right),
$$

where the fields $b_{i}$ are massless bottom-like fields. Additional $N_{L}-1$ massive $W^{ \pm}$-bosons and one massive Z-boson as well as $N_{L}\left(N_{L}-2\right)$ massless vector bosons emerge. All these vector bosons will not play a role in our discussion, which will become clear in the next section. As an important fact we have $N_{L}-1$ charged Goldstone bosons $G^{ \pm}$, which become the longitudinal components of the $N_{L}-1$ massive $W^{ \pm}$-bosons above. In total we end up with the generalized Higgs Lagrangian $\mathcal{L}_{I}$ :

$$
\begin{aligned}
\mathcal{L}_{I}= & \frac{1}{2} \frac{g_{t}^{2} N_{c}}{G}\left(H H+G^{0} G^{0}+2 \sum_{i=1}^{N_{L}-1} G_{i}^{+} G_{i}^{-}\right) \\
& -\frac{g_{t}}{\sqrt{2}} H \bar{t} t+i \frac{g_{t}}{\sqrt{2}} G^{0} \bar{t} \gamma^{5} t+g_{t} \sum_{i=1}^{N_{L}-1}\left(G_{i}^{+} \bar{t} L b_{i}+G_{i}^{-} \bar{b}_{i} R t\right) .
\end{aligned}
$$

Now it seems simple to expand the gap equation and the boson self-energies in $1 / N_{c}$ and $1 / N_{L}$. Nevertheless it is not clear what " $1 / N_{L}$ " means. In the following " Order $\left(N_{L}\right)^{n}$

" means that there are $\mathrm{n}$ summations over the Goldstone boson degrees of freedom and it does not mean that the factor $N_{L}$ appears n times. The reason is that the Higgs boson must also be included with various combinatorial factors and leads usually to a factor $\left(N_{L}+1\right)^{n}$ instead of $\left(N_{L}\right)^{n}$.

In the next section we discuss the lowest order in $1 / N_{c}$ and $1 / N_{L}$, which means order $\left(1 / N_{c}\right)^{n_{1}} \cdot\left(1 / N_{L}\right)^{n_{2}}$ with $n_{1}+n_{2}=0$, where each boson line contains $1 / N_{c}$ and each flavor summation $N_{L}$.

\section{The cancellation of quadratic divergencies}

Our aim is now to establish the gap equation in lowest order. Due to flavor expansion it contains also top self-energies with exchange of one or more Higgs or Goldstone bosons 
like in Fig. 3, where the two boson lines give $\left(1 / N_{c}\right)^{2}$. Surprisingly there are less than two flavor summations. Let us consider the first diagram. If the outer boson line is a $G^{+}$, the fermion which moves between the outer and inner boson line is a b-quark. Therefore the inner boson line has to be a $G^{-}$but cannot be a $H$ or $G^{0}$. That is the reason why there is only one flavor summation. The second diagram has even no flavor summation. One comes to the conclusion that only a one Higgs or one Goldstone boson exchange diagram contributes to the lowest order gap equation. From that it is also not correct to add this one boson diagram to the tadpole in Fig. 1 because the gap equation would generate the first diagram of Fig. 3 in a recursive way. One finds that the gap equation must have the form of Fig. 4. The full top self-energy is denoted by a double circle, which does not appear in the boson exchange diagram to guarantee the absence of diagrams of Fig. 3. On the contrary the tadpole diagram is iterated because it contains the full top self-energy.

It is interesting that the new diagram is not of the order $g_{t}^{2}$ as one would expect with regard to the Standard Model. The boson propagator contains a factor $\zeta^{-1}$ which is proportional to $g_{t}^{-2}$ and cancels the $g_{t}^{2}$ coming from the vertices. This is not surprising and expresses the fact that form factors are of the order one. On the contrary vector bosons would contribute proportional to $g_{1}^{2}$ or $g_{2}^{2}$ which really is a higher order effect.

Unfortunatly the equations of Fig. 4 are a system of coupled integral equations. But we are mainly interested in the leading quadratic divergencies of the tadpole graph. It is therefore possible to combine both equations in the following way: We substitute the full double circle in the second equation by the geometrical series of the first equation denoted with the empty double circle. But the last graph of the first equation cannot be put in the tadpole twice or more because the fermion loop of the tadpole graph would be finite due to more than four outer boson lines. The gap equation for the leading order divergencies is shown in Fig. 5, which now contains the expected boson tadpole diagrams as in the Standard Model yielding the second contribution of eq. (11).

In contrast to the usual Standard Model the boson tadpoles contain an effective vertex $\Gamma$ with three outer boson lines. As one can easily verify, in the leading logarithmic divergency there is a simple relation between $\Gamma$ and the corresponding Standard Model tree vertex $\Gamma_{S M}$ independent of the kind of the outer boson lines:

$$
\Gamma=\zeta\left(\frac{2 m_{t}}{M_{H}}\right)^{2} \Gamma_{S M}
$$

In the tadpole diagram the $\zeta$ of the three Higgs vertex is cancelled by the $\zeta^{-1}$ coming from the boson propagator. Therefore the boson contribution can simply be calculated by using 
the usual Standard Model formula multiplicated by a factor $\left(2 m_{t} / M_{H}\right)^{2}$. The two first contributions of eq. (1) in the Standard Model give in top condensation models:

$$
\left(4 m_{t}^{2}-M_{H}^{2}\left(\frac{2 m_{t}}{M_{H}}\right)^{2}\right) \Lambda^{2}
$$

which exactly disappear. The reason for the cancellation is not the relation $M_{H}=2 m_{t}$ which we have not demanded but is the fact that the Higgs is a $\bar{t} t$-boundstate and only couples to the top quark.

The calculation above is not yet a proof to all orders of perturbation theory, but it seems that there can be a cancellation mechanism for quadratic divergencies without Supersymmetry. Even more, this cancellation mechanism depends on the group structure of the Standard Model. If we replace

$$
S U(3)_{c} \times S U(2)_{L} \times U(1)_{Y} \rightarrow S U\left(N_{c}\right)_{c} \times S U\left(N_{L}\right)_{L} \times U(1)_{Y}
$$

the quadratic divergencies read

$$
\left(\frac{N_{c}}{3} 4 m_{t}^{2}-\frac{N_{L}+1}{3} M_{H}^{2}\left(\frac{2 m_{t}}{M_{H}}\right)^{2}\right) \Lambda^{2}
$$

and only disappear, if

$$
N_{c}=N_{L}+1
$$

It is interesting that even the Standard Model has such a structure. If this mechanism works, it would mean that a Standard Model with the wrong group structure would already break at the Grand Unification scale. But if physics prefers a wide scale hierarchy for example to lower the energy, the Grand Unified gauge group will prefer to break to the usual Standard Model. In this sense top condensation could explain this unusual breaking of the Grand Unified Theory, which is not understood up to now.

So far we have not included the influence of the vector bosons. As shown in eq. (四) they also provide quadratic divergencies in the Standard Model. In contrast to the Goldstone bosons their couplings to the top quark are not of the order one but proportional to $g_{1}$ or $g_{2}$. They give higher order contributions to our tadpole diagrams of Fig. 5 and to the boson self-energy diagrams of Fig. 2, which is shown in Fig. 6. All corrections can be understood as a redefinition of the top-Yukawa-coupling. Therefore the quadratic divergencies of the radiative corrections should also disappear. Otherwise gauge bosons would provide a hierarchy problem in contradiction to 't Hoofts naturalness principle. 


\section{The lowest order gap equation}

In the Standard Model the quadratic divergencies lead to a big difference between bare and renormalized masses as mentioned above. In top condensation models the appearance of quadratic divergencies has a slightly different meaning. If $\Lambda$ in eq. (6) is many orders of magnitude larger than the weak scale, the $m_{t} / \Lambda$-ratio strongly depends on the choice of the four-fermion-coupling constant $G$. For a natural range of $G m_{t} / \Lambda$ is of the order one so that very small values of $m_{t} / \Lambda$ need an extreme fine-tuning of $G$.

Including flavor expansion and after calculating the quadratic and logarithmic divergencies of both graphs in Fig. 5 the gap equation has the form:

$$
1=\frac{2 G}{(4 \pi)^{2}}\left(\Lambda^{2}-m_{t}^{2} \ln \frac{\Lambda^{2}}{m_{t}^{2}}\right)-\frac{2 G}{(4 \pi)^{2}}\left(\Lambda^{2}-\frac{1}{2} M_{H}^{2} \ln \frac{\Lambda^{2}}{m_{t}^{2}}\right)
$$

or

$$
1=\frac{2 G}{(4 \pi)^{2}}\left(\frac{M_{H}^{2}}{2}-m_{t}^{2}\right) \ln \frac{\Lambda^{2}}{m_{t}^{2}} .
$$

The situation changes drastically. Even for a very large value of $\Lambda$ there is no fine-tuning and the relation between $G$ and $m_{t}$ depends only slightly on the cutoff $\Lambda$. If the Higgs mass is approximately twice the top mass we get

$$
1=\frac{2 G}{(4 \pi)^{2}} m_{t}^{2} \ln \frac{\Lambda^{2}}{m_{t}^{2}} .
$$

Putting in the Planck mass $\Lambda_{\text {Planck }}$ this yields

$$
\frac{2}{(4 \pi)^{2}} \ln \frac{\Lambda_{\text {Planck }}^{2}}{m_{t}^{2}} \approx 1
$$

and

$$
G^{-1} \approx m_{t}^{2}
$$

This is a surprising result. The scale of the interaction which establishes the $\bar{t} t$-boundstate is of the same order as the top mass itself. The new interaction could be the Standard Model itself, or more precisely the top-Higgs-interaction with a big top-Yukawa-coupling. This is precisely Nambu's bootstrap idea [9], where the Higgs is built up by interacting with its own constituents.

Nevertheless one has to be careful about equation eq. (19). We have only used the leading

divergencies which only provide valid results for the quadratic divergencies. We must 
further consider that the flavor expansion is broken by asymmetries between the Higgs and the Goldstone bosons coming from the different masses. From that one has to expect that the lowest order of the flavor expansion provides logarithmic divergent radiative corrections to the Goldstone boson self-energies which unfortunately make them massive. Flavor expansion only remains a tool to show the cancellation of quadratic divergencies and cannot be used to calculate logarithmic corrections to the Higgs and Goldstone boson masses.

\section{Discussion}

Motivated by the similarity between the Veltman condition applied to the usual Standard Model and the additional condensate condition for a top mode Standard Model we have studied a cancellation mechanism for quadratic divergencies in top condensation models. In the zeroth order of the usual $1 / N_{c}$-expansion a cancellation of quadratic divergencies cannot appear due to the missing Higgs and Goldstone boson contributions. Using the Goldstone boson degrees of freedom as a further expansion parameter we could show that the quadratic divergencies cancel to lowest order without demanding any mass relation and independent of the final values of the top and the Higgs mass. This is based on a natural relation between top quark and Higgs boson in top condensation models similar to the relation between fermions and bosons in supersymmetry. It is difficult and maybe impossible however to show such a Quasi-Supersymmetry between a fundamental and a composite particle in any order of perturbation theory. Nevertheless it is interesting that the cancellation depends on the group structure of the weak and strong interaction. This can be understood in the following way: We first have to take into account that the Higgs and Goldstone bosons consists of two constituents. One can therefore replace each boson line of a Feynman graph by two fermion lines. The interaction between any two fermions is represented by the small distance of the lines. In this way the gap equation of fig. 5 can be translated into the form of fig. 7. The outer top lines have an explicite color index a. One can easily see that the first graph has neither a color nor a flavor summation. In contrast the second graph contains a summation over the $N_{L}+1$ flavor degrees $t_{R}^{a}, t_{L}^{a}, b_{1, L}^{a}, \ldots, b_{N_{L}-1, L}^{a}$ $\left(N_{L}=2\right.$ in fig. 7$)$ and an additional factor $(-1)$ due to the extra fermion loop. The bosons are color neutral so that the inner fermions have the corresponding color quantum number and no color summation appears. Even more there is a factor $1 / N_{c}$ : After insertion of a four-fermion-vertex between the outer and inner fermion line there is no closed color loop but the vertex contains a factor $1 / N_{c}$. We therefore conclude that the second graph has an additional factor $-\left(N_{L}+1\right) / N_{c}$. So in lowest order the quadratic divergencies cancel 
for $1-\left(N_{L}+1\right) / N_{c}=0$. The cancellation is therefore a consequence of the same number of color and flavor degrees in top condensation models. This symmetry between degrees of freedom seems to protect top condensation models against the presence of quadratic divergencies, at least in lowest order.

We have also seen that this cancellation cannot be destroyed by the vector boson contributions due to 't Hoofts naturalness principle. As one can simply verify following our procedure, the quadratic divergencies of the boson self-energies also cancel in the same way as the tadpoles. As above one has to consider the corresponding Standard Model graphs but must insert effective boson vertices.

We have further shown that the resulting gap equation provides a top mass of the order of the four-fermion-interaction scale because of the missing quadratic divergencies. Since the four-fermion-interaction could be the exchange of Higgs bosons itself, the bootstrap scenario could be a natural consequence of our cancellation mechanism. Nevertheless the experimentell limits on the $\rho$-parameter in the Standard Model seems to give a too low top mass and top-Yukawa-coupling, which is not sufficient to establish a condensation. In top condensation however the top mass dependence of the $\rho$-parameter changes and admits larger top masses than in the Standard Model [10]. Our model could therefore be a real alternative to Supersymmetry but is much more simple. Its only disadvantage is the difficulty in calculating dynamical processes beyond the bubble approximation but should not prevent us from moving on in this direction.

Acknowledgements: I would like to thank M. Beneke, R. Bönisch, M. Lindner, B. Stech and L. Vergara for many useful discussions. 


\section{References}

[1] G. 't Hooft, Cargese, NATO (1979) 135.

[2] H. P. Nilles, Phys. Rep. 110 (1984) 1.

[3] R. Decker and J. Pestieau, UCL-ITP-79-19 (1979);

M. Veltman, Acta Phys. Pol. B12 (1981) 437.

[4] Y. Nambu, EFI 85-86 (1985) and EFI 88-39 (1988).

[5] W. A. Bardeen, C. T. Hill and M. Lindner, Phys. Rev. D41 (1990) 1647.

[6] C. T. Hill, Phys. Lett. B266 (1991) 419;

R. Bönisch, Phys. Lett. B268 (1991) 394;

M. Lindner and D. Ross, Nucl. Phys. B370 (1992) 30.

[7] A. Hasenfratz et al. , Nucl. Phys. B365 (1991) 79;

U. Ellwanger and L. Vergara, Nucl. Phys. B398 (1993) 52;

M. Lindner, Int. Journal of Mod. Phys. A8 (1993) 2167.

[8] Y. Nambu and G. Jona-Lasinio, Phys. Rev. 122 (1961) 345.

[9] Y. Nambu, EFI 90-46 (1990); EFI 90-69 (1990); EFI 90-89 (1990).

[10] A. Blumhofer and M. Lindner, Nucl. Phys. B407 (1993) 173. 


\section{Figure Captions}

Figure 1: The gap equation in leading order $1 / N_{c}$. The empty dot is the one-particleirreducible self-energy. One gets the full dot, which represents the full propagator, by summing up the empty dots.

Figure 2: Higgs and Goldstone boson self-energies in leading order $1 / N_{c}$.

Figure 3: Higher order contributions to the top self-energy in color and flavor expansion.

Figure 4: The system of gap equations in leading order $1 / N_{c}$ and $1 / N_{L}$. The full double circle is the full top propagator, which can be found by summing up the empty double circle. The full and empty dots are auxiliary objects and the full dot is the sum of the empty one.

Figure 5: The gap equation in leading order $1 / N_{c}$ and $1 / N_{L}$ and in the leading divergencies.

Figure 6: Vector boson contributions to the gap equation.

Figure 7: A form of the gap equation of Fig. 5, where the constituents are visible. It shows the group structure dependence of the cancellation mechanism. 
This figure "fig1-1.png" is available in "png" format from: http://arxiv.org/ps/hep-ph/9311282v1 
This figure "fig1-2.png" is available in "png" format from: http://arxiv.org/ps/hep-ph/9311282v1 
This figure "fig1-3.png" is available in "png" format from: http://arxiv.org/ps/hep-ph/9311282v1 